\title{
Dairy products as a source of iodine in the UK, and consumers' perceptions
}

\author{
M. Bouga, M.E.J. Lean and E. Combet \\ Human Nutrition, School of Medicine, Dentistry and Nursing, College of Medical, Veterinary and Life Sciences, \\ University of Glasgow, G31 2ER.
}

Dairy product consumption has been linked to health benefits, from lowering blood pressure and LDL cholesterol, to preventing type II diabetes and obesity ${ }^{(1)}$. Milk is also the main source of iodine in the $\mathrm{UK}^{(2)}$, a country unexpectedly re-categorised as iodine insufficient $^{(3)}$. Dairy consumption tends to decrease with age ${ }^{(4)}$ and a large proportion of women of childbearing age avoid dairy despite their nutritional importance. This study investigated the consumption and perceptions (knowledge and awareness) of the nutritional value of dairy products in the UK.

A validated questionnaire, informed by the theory of planned behaviour, was disseminated online and in the community (through social media, fora, snowball recruitment in public spaces) to English-speaking UK residents aged 14 years and over, between June and November 2016. The drivers for choosing dairy products were rated using 5-point Likert scales (1: not at all, 5: very much). Iodine intake from dairy products was estimated using a validated food frequency questionnaire ${ }^{(5)}$. Descriptive statistics were expressed as median and interquartile range (IQR) for continuous non-parametric data and as frequencies (n) and percentages (\%) for categorical data (SPSS version 21.0, IBM Corporation). Groups were compared with Mann-Whitney U tests.

The median age of the respondents $(\mathrm{n}=2040)$ was 26 years $($ IQR $21-39)$ with $5 \%(\mathrm{n}=111)$ identifying as dairy avoiders $($ ovovegetarians or vegans).

Total dairy products consumption was 260 g per day (IQR 124-446), with milk contributing 65\% (IQR 39-80) of total dairy consumption based on weight consumed. The median dairy products servings per day were 2.2 (IQR 1.2-3.5). The median iodine intake from dairy products was $85 \mu \mathrm{g} /$ day (IQR 44-147), with $45 \mu \mathrm{g} /$ day (IQR 10-83) derived from milk. Almost half $(45 \%, \mathrm{n}=922)$ of the participants did not achieve the recommendation of 2 servings/day for dairy products ('low consumers'). The main reported factors identifiable as barriers to dairy consumption (Likert scale $4 \& 5)$ in 'low consumers', included taste $(78 \%, \mathrm{n}=716)$, freshness $(60 \%$, $\mathrm{n}=550)$ and texture $(55 \%, \mathrm{n}=508)$. 'High consumers' of dairy products $(54 \%, \mathrm{n}=1104)$ had significantly higher iodine intake compared to 'low consumers' (137 $\mu$ g/day (IQR 102-195) vs $41 \mu \mathrm{g} /$ day (IQR 13-61), p < 0.01) - drivers (Likert scale 4\&5) for consumption included taste $(89 \%, \mathrm{n}=980)$, freshness $(71 \%, \mathrm{n}=785)$ and smell $(61 \%, \mathrm{n}=676)$.

Dairy consumption behaviours were rarely influenced by a healthcare professional (GP, nutritionist, dietician, other) (35\% in agreement, $n=716)$, or health expert in the media $(16 \%$ in agreement, $n=322)$. Sustainability of dairy food production was a concern to 'low consumers', with $41 \%(n=376)$ perceiving a negative impact on the environment, against $19 \%(n=218)$ of the 'high consumers'. From a health perspective, $25 \%(\mathrm{n}=233)$ of 'low consumers' believed that dairy products should be avoided to limit saturated fats intake.

The NDNS has shown decreased intake of milk from 1997 to $2008^{(6)}$, and half of the population is not meeting the recommended two dairy portions a day. Low dairy intake is likely to impact on iodine intake, and also other nutrients, such as calcium and vitamin B12; it is therefore important that nutritionally-sound alternatives (such as plant-based milk and fortified products) are made available to the rising population excluding dairy from their diet. New concerns, such as sustainability, and environmental impact, combined to poor knowledge of the nutritional composition of dairy products must be considered when developing public health interventions aiming to increase dairy consumption.

1. Tunick MH, Van Hekken DL. J Agric Food Chem. 2014

2. Bates B, Lennox A, et al. National Diet and Nutrition Survey Headline results from Years 1 and 2 (combined) of the Rolling Programme (2008/ 2009-2009/10). 2011.

3. Vanderpump MPJ, Lazarus JH, et al. Lancet. 2011;377(9782):2007-2012.

4. Dror DK, Allen LH. Nutrition reviews. 2014;72(2):68-81.

5. Combet E, Lean MEJ. Journal of Human Nutrition and Dietetics. 2014;27(6):599-605.

6. Whitton C, Nicholson SK, et al. British Journal of Nutrition. 2011;106(12):1899-1914. 